\title{
GENETIC CONTROL OF SOMATIC CELL FUSION IN A MYXOMYCETE
}

\author{
HUBERT LING and MILDRED LING \\ Department of Biological Sciences, University of Delaware, Newark, Delaware 19711
}

Received 29.i.73

\begin{abstract}
SUMmary
Somatic cell fusion in the myxomycete Didymium iridis is controlled by at least 11 loci. Each locus has a pair of alternate alleles and diploid cells (plasmodia) must be phenotypically identical in order to fuse freely with each other. Cells with unknown fusion alleles can be tested against genetically defined testers. Thus identification of alleles at known fusion loci is relatively easy. The study of new fusion alleles is much more complex since no testers exist. However, $\mathrm{F}_{1}$ 's from a given cross can be analysed simultaneously for several new fusion loci without resorting to an additional breeding programme. Theoretical considerations for this procedure are presented. Of the 11 loci, seven have been identified as strong loci; these loci do not permit any plasmodial fusion. Four fusion loci have been identified as weak loci; they allow a partial, temporary fusion which is terminated within 2 to 3 minutes by cytoplasmic coagulation. Possible biochemical mechanisms in these cell recognition phenomena are discussed.
\end{abstract}

\section{InTRODUCTION}

Somatic cell fusion is known to occur only in a relatively small number of organisms. Asexual fusion between genetically identical cells of mammalian skeletal muscle, fungal hyphae and true slime mould plasmodia, occurs as a normal part of growth and development. The occurrence of somatic cell fusion in these organisms appears to be an exception to the general rule that once somatic cells are formed they do not fuse with other cells.

Asexual cell fusion between cells of different genotype (the production of heterokaryons) is even more rare. Although such fusion can be accomplished in the laboratory with a wide range of cells, the conditions conducive to such fusion rarely occur in nature (cf. Harris, 1970; Poole, Howell and Lucy, 1970; Power, Cummins and Cocking, 1970).

General lack of asexual fusion between genetically dissimilar cells is expected since even at the unicellular level organisms have developed elaborate mechanisms to distinguish "self" from foreign " non-self".

The fungi are not particularly well suited to genetic studies on cell fusion because of their small size but numerous studies have been made on heterokaryon incompatibility in several fungi: Aspergillus glaucus (Jones, 1965), Neurospora crassa (Wilson and Garnjobst, 1966), Podospora anserina (Bernet, 1967; Fincham and Day, 1971), Aspergillus sp. (Caten, 1971).

In the myxomycetes, however, both heterokaryon studies and studies on the genetic control of cell fusion can be done since the plasmodium is motile, coenocytic and macroscopic (Collins and Clark, 1968; Ling and Collins, 1970 a, b; Collins and Clark, 1973).

Incompatibility studies in Didymium iridis have been carried out for the past 11 years (Alexopoulos and Zabka, 1962; Collins, 1963; Mukherjee, 
1965). It was immediately apparent that a complex genetic system controls somatic cell fusion and cytoplasmic mixing between two diploid plasmodia. Even with the same parents, plasmodia only fuse with a small percentage of their siblings. The basic genetic principles were laid down when three loci, $C, D$ and $E$, were proposed which permit fusion between individuals with identical phenotypes (Collins and Clark, 1966). Later studies indicated that numerous other loci also control the fusion process.

It was thought at first that all fusion loci simply prevented cell fusion. However, it has recently been shown (Collins and Clark, 1973; Upadahyaya and Ling, 1972) that several of the fusion loci allow temporary fusion of two plasmodia. This temporary fusion is terminated within a few minutes by coagulation of the area where cytoplasmic mixing has occurred. The affected area loses pigment and is termed a clear zone. The basic principle is that two plasmodia must be phenotypically identical in order for them to fuse and become a single entity. Phenotypic differences at certain loci (termed weak loci) do not prevent temporary cell fusion but do prevent extensive exchange of nuclei and protoplasm since protoplasmic coagulation always takes place within 2 or 3 minutes. In this paper we will summarise the methods which are used to assign phenotypes to representative plasmodia and to thus determine the genotypes of haploid amoebal clones. Methods are presented which demonstrate how to detect new fusion loci and how to assign genotypes to material produced in 3-locus and 4-locus test crosses. In addition preliminary studies on the functions of the known fusion loci are discussed and nomenclature revisions are presented.

\section{Materials AND MEthods}

The materials and methods used here are similar to those detailed by Collins and Clark (1968), and Ling and Collins (1970a). To summarise, a diploid parent plasmodium can undergo meiosis to form haploid spores. A single spore germinates to form a haploid myxamoeba which soon produces a large population of genetically identical myxamoebae (a clone). Myxamoebae can also act as gametes. Mating is controlled by multiple alleles (e.g. $A^{1}, A^{2}, A^{3}, A^{4}, A^{4 m-1}, A^{5}, A^{6}$ ) at the mating locus " $A$ " (Collins, 1963; Collins and Ling, 1964). $F_{1}$ plasmodia are formed when clones of different mating types are mixed together. $F_{1}$ plasmodia may then be allowed to sporulate and haploid $\mathrm{F}_{1}$ clones can be established. Diploid $\mathrm{F}_{2}$ plasmodia are produced by mixing compatible $\mathrm{F}_{1}$ clones together.

The alleles at established fusion loci can be inferred by test fusing the plasmodium in question to one with a known fusion phenotype. Fusion and uninhibited cytoplasmic mixing indicates that the two plasmodia are phenotypically identical at all the fusion loci. Lack of vigorous and continuous cytoplasmic mixing means that phenotypic differences occur at one or more loci. The existence of previously undetected fusion loci is determined by crossing two clones and analysing the progeny as shown diagrammatically in fig. 1. Figs. 2, 3 and 4 show how fusion tests appear under the binocular dissecting microscope.

In this study the haploid clones P4 (mating type $A^{4 m-1}$ ) and $\mathrm{H} 7$ (mating type $A^{2}$ ) will be referred to as parental clones. Clones of the Hon. 1 strain are prefaced with an $\mathrm{H}$, those from Pan. 1 strain are prefaced with a $\mathrm{P}$. Hybrid clones are labelled HP. When the parental clones were crossed the 
diploid $\mathrm{F}_{1}$ plasmodium $\mathrm{P} 4 \cdot \mathrm{H} 7$ was produced. A dot between two symbols represents a diploid plasmodium, a dash represents a haploid clone. The $F_{1}$ plasmodium was allowed to sporulate and $F_{1}$ spores were isolated individually by hand with a fine needle. Isolations are done at $45 \times$ or $90 \times$. Since meiosis occurs in the maturing spores, the myxamoeba which are produced upon spore germination are haploid. The $F_{1}$ clones were backcrossed to the parental clone with the compatible mating type and the resultant plasmodia were fused among themselves to see how many different fusion types existed (fig. 1).

Generation

Parent $(2 \mathcal{N})$

Gamete $(\mathcal{N})$

$\mathbf{F}_{1}(2 \mathcal{N})$

$\mathbf{F}_{1}$ Gametes $(\mathcal{N})$

Backcross Plasmodia $(2 \mathcal{N})$
Hon. 1 Plasmodium

Sporulation and Meiosis

Pan. 1 Plasmodium

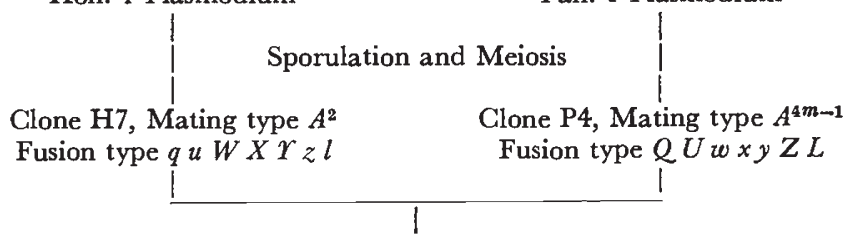

Plasmodium $\mathrm{P} 4 \cdot \mathrm{H} 7$,

Phenotype $=Q U W X Y Z L$

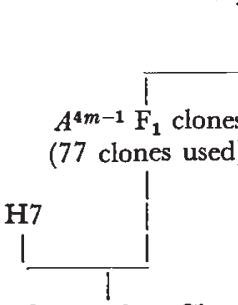

Set No. 1, backcrossed to Glone H7,

77 plasmodia of 16 fusion classes

(Segregation at loci $Q U Z L$ )

\section{Meiosis}
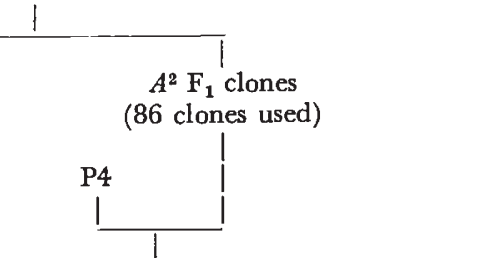

Set No. 2, backcrossed to Glone P4, 86 plasmodia of 8 equal fusion classes (Segregation at loci $W X Y$ )

FIG. 1.-Determination of the number of alleles segregating out in two sets of backcrosses.

To determine how many fusion classes exist in a given set of backcrosses, one plasmodium is chosen as a tester and attempts are made to fuse this plasmodium to the other plasmodia. Plasmodia that fuse with the first tester belong to that fusion class. Members of a fusion class have been found to fuse with each other in all combinations. Plasmodia that do not fuse to the first tester belong to other fusion classes. A second tester is chosen from these plasmodia and run against all remaining plasmodia. This process is repeated until all plasmodia fuse with a given tester. A plasmodium that does not fuse with any other is itself a representative of a separate fusion class. The backcross method has been shown to provide accurate results without resorting to much more tedious $\mathrm{F}_{2}$ analyses (Ling, 1971b).

\section{Results}

(i) Previous studies with the Hon. 1 and Pan. 1 strain of Didymium iridis

Studies with the Hon. 1 strain indicated that at least five loci, $C$ to $G$, controlled somatic fusion (Collins and Clark, 1968), and six fusion loci, $Q$ to $V$, were reported in the Pan. 1 strain of D. iridis (Ling and Collins, $1970 a$ ). When hybrids between Hon. 1 and Pan. 1 were made it was 
demonstrated that at least 11 fusion loci control cytoplasmic mixing in D. iridis (Collins and Ling, 1972). It is believed that many of the Hon. I fusion loci are identical to those reported in the Pan. 1 strain. However, at the present time we do not know which locus is equivalent to which in these two strains. The 1972 report is summarised diagrammatically in fig. 1 .

Fig. 1 shows that the $A^{2} \mathrm{~F}_{1}$ 's that mated with the $A^{4 m-1}$ parent (P4) produced plasmodia that fell into 8 equal fusion classes. Classes of 10,10 , $9,13,8,14,11$ and 11 were produced. The $A^{4 m-1} \mathrm{~F}_{1}$ clones that mated with the $A^{2}$ parent $(\mathrm{H} 7)$ produced plasmodia that fell into 15 unequal fusion classes: $10,8,12,12,5,4 ; 4,5,3,3,3,3,1,1$, and 3 . These latter results are believed to reflect linkage patterns which are currently being investigated. Although only 15 fusion classes were recovered in this set, data presented in this paper indicate strongly that theoretically 16 fusion classes were possible in backcross set No. 1. The occurrence of one or more groups of linked alleles in this backcross set would make recovery of 16 fusion classes difficult unless a very large sample were studied. To simplify the results we will hereafter assume that 16 fusion classes are recoverable.

The existence of 16 fusion classes in set No. 1 was explained genetically by assuming that clone $\mathrm{H} 7$ was recessive at four fusion loci which had dominant alleles in P4. Eight fusion classes in backcross set No. 2 were explained by assuming clone $\mathrm{P} 4$ had recessive alleles at three previously undetermined fusion loci named $W, X$ and $r$ which were dominant in $\mathrm{H} 7$. From previous studies clone $\mathrm{P} 4$ was determined to be dominant at all six fusion loci known from the Pan. I strain ( $R S T U V$ ). By using the information above we can assign a genotype of $Q R S T U V w x y$ to clone $\mathrm{P} 4$ at the fusion loci. It has also been determined that $\mathrm{H} 7$ was $q R S T u V$ at the fusion loci of the Pan. 1 strain (Collins and Ling, 1972). Two more recessives, $z l$ were postulated for $\mathrm{H} 7$, with $\mathrm{P} 4$ possessing dominants at these loci. The resulting genotypes for the two parental clones are thus $\mathrm{P} 4=Q R S T U V w \times y Z L$ (mating type $A^{4-1}$ ),

$$
\mathrm{H} 7=q R S T u V W X Y z l \text { (mating type } A^{2} \text { ) }
$$

(Collins and Ling, 1972). Since parental clones $\mathrm{P} 4$ and $\mathrm{H} 7$ both possess dominant alleles at fusion loci $R S T$ and $V$ these loci will generally be ignored in this study.

The 1972 report showed that numerous fusion classes were produced in each backcross set due to a theoretical segregation of fusion loci. However, the genotypes and phenotypes of the tester plasmodia remained unknown. A particular handicap was the lack of genetically defined material at the newly identified loci $W, X, \Upsilon, Z$ and $L$. This report shows how representative tester clones were genetically defined at these new loci.

\section{(ii) Backcross set No. 1}

Experimentally the 77 plasmodia of backcross set No. 1 (table 1) were tested until each fell into a fusion class. One healthy plasmodium from each fusion class was chosen as the tester plasmodium to represent that class in subsequent tests. The $A^{4 m-1} \mathrm{~F}_{1}$ 's which were used to create the tester plasmodia were referred to as tester clones. Since parental clone H7 has a $q u z l$ genotype, genotypic variation in the tester $F_{1}$ clones at these loci results in the 16 phenotypes of the tester plasmodia and thus 16 different fusion classes. The 16 expected fusion classes are shown in table 1. Segregation at the $W, X$ and $Y$ fusion loci also takes place in the $\mathrm{F}_{1}$ 's but phenotypic 
expression in plasmodial testers $1-16$ is prevented due to dominants at these loci in clone $\mathrm{H} 7$.

\section{(iia) Allelic determinations at loci $\mathrm{Q}$ and $\mathrm{U}$}

The 16 fusion classes can theoretically be divided into four groups of four fusion classes, each, based on their alleles at $Q$ and $U$ (i.e. $Q U, Q u$, $q U, q u)$. Determination of alleles at these two loci is relatively simple since tester material of known genotype at these two loci was available from previous studies.

The test to determine the allele at the $Q$ locus in an $F_{1}$ clone consists of crossing the $A^{4 m-1}$ clone with clones P44 (mating type $A^{3}$ ) and P50 (mating type $A^{3}$ ) to give plasmodia as follows:

$$
\mathrm{F}_{1} \cdot \mathrm{P} 44=\frac{A^{4 m-1}}{A^{3}} \frac{Q ?}{Q} \quad \frac{U ?}{U} \quad \frac{Z ?}{Z} \quad \frac{L ?}{L} \quad \frac{W ?}{w} \quad \frac{X ?}{x} \quad \frac{r ?}{y}
$$

phenotype $=Q U Z L\left(W X Y\right.$ determined by alleles of $\mathrm{F}_{1}$ clone $)$

$$
\mathrm{F}_{1} \cdot \mathrm{P} 50=\frac{A^{4 m-1}}{A^{3}} \quad \frac{Q ?}{q} \quad \frac{U ?}{U} \quad \frac{Z ?}{Z} \quad \frac{L ?}{L} \quad \frac{W ?}{w} \quad \frac{X ?}{x} \quad \frac{r ?}{y}
$$

phenotype $=U Z L\left(Q W X r\right.$ determined by $\mathrm{F}_{1}$ clone $) . \quad$ The genotype of the $F_{1}$ clone being tested is given above the line and the genotype of the second clone P44 or P50 is listed below the lines. A "? " indicates that the allele at this locus is unknown.

The genotypes of clones P44, P50, P69 and other tester clones are discussed in Ling (1971 $a$ ) and in Ling and Collins (1970a,b). The phenotype of the plasmodium $F_{1} \cdot P 50$ at the $Q$ locus is determined by the $F_{1}$ clone. Hence if $F_{1} \cdot P 44$ is test fused against $F_{1} \cdot P 50$, a fusion will result if the $F_{1}$ has a dominant $Q$ allele, and a non-fusion indicates the $F_{1}$ is recessive.

Similarly the $\mathrm{F}_{1}$ clones were tested at the $U$ locus. $\mathrm{F}_{1}$ clones were crossed to clones P44 and P69 and test fused to each other. The genotype of P69 is $Q u Z L w x y$ so that the phenotype of $\mathrm{F}_{1} \cdot \mathrm{P} 69$ is $Q U ? Z L(W X r$ determined by $F_{1}$ clone). Fusion indicates that the $F_{1}$ has the dominant allele at $U$ and non-fusion indicates a recessive.

\section{(iib) Allelic determination at the new loci $\mathrm{Z}$ and $\mathrm{L}$}

Once the $A^{4 m-1}$ tester clones have been determined at fusion loci $Q$ and $U$, four groups with four classes per group (i.e. $Q U, Q u, q U, q u$ ) have been established. The four fusion classes in each group are formed by segregation of the new fusion loci $Z$ and $L$ (table 1). Determination of the alleles at loci $Z$ and $L$ for each of the 16 tester clones cannot be done systematically but must be done piecemeal by using several different sets of testers. The numerous tests necessary to do this will not be described in detail. Basically, the $\mathrm{F}_{1}$ plasmodium $\mathrm{H} 7 \cdot \mathrm{P} 4$ is known to have a $Q U Z L$ phenotype because of the genotypes assigned to $\mathrm{H} 7$ and $\mathrm{P} 4$. This plasmodium was thus used to test for clones dominant at both $Z$ and $L$. Plasmodia were available from the Hon. 1 strain that possessed a $z l$ phenotype and tests for recessives at both $z$ and $l$ were possible. Since $Z$ and $L$ are newly described fusion loci the genotype assignment of one clone to $z L$ and to another of $Z l$ is arbitrary. 
It is, however, necessary to perform tests to ensure that the $z L$ or $Z l$ in each of the four groups is equivalent.

(iii) Backcross set $\mathcal{N}$ o. 2: Allelic analyses at the new loci $\mathrm{W}, \mathrm{X}$ and $\mathrm{Y}$

In this set of eight fusion classes, fusion class No. 17 ( $W X Y$ ) can easily be identified by fusion tests to the $\mathrm{F}_{1}$ plasmodium $\mathrm{H} 7 \cdot \mathrm{P} 4$ since fusion class 17 and $\mathrm{H} 7 \cdot \mathrm{P} 4$ have the same phenotype, $W X Y$ (table 1). Fusion class No. 24

\section{TABLE 1}

Production of fusion classes due to segregation of alleles in two sets of backcrosses

A. The $A^{4 m-1} \mathrm{~F}_{1}$ 's backcrossed to clone $\mathrm{H} 7$ (set No. 1)

$\begin{array}{cccc}\text { Fusion class } & \text { Genotype } & \text { Fusion class } & \text { Genotype } \\ \text { (1) } & Q U Z L & (9) & q U Z L \\ (2) & Q U Z l & (10) & q U Z l \\ (3) & Q U z L & (11) & q U z L \\ (4) & Q U z l & (12) & q U Z l \\ (5) & Q u Z L & (13) & q u Z L \\ (6) & Q u Z l & (14) & q u Z l \\ (7) & Q u z L & (15) & q u z L \\ (8) & Q u z l & (16) & q u z l\end{array}$

B. The $A^{2} \mathrm{~F}_{1}$ 's backcrossed to clone P4 (set No. 2)

$\begin{array}{cccc}\text { Fusion class } & \text { Genotype } & \text { Fusion class } & \text { Genotype } \\ (17) & W X Y & (21) & w X Y \\ (18) & W X y & (22) & w X y \\ (19) & W x Y & (23) & w x Y \\ (20) & W x y & (24) & w x y\end{array}$

$(w x y)$ can easily be found by test fusions with $\mathrm{P} 44 \cdot \mathrm{P} 4$ with phenotype $w x y$. Tester plasmodium $\mathrm{HP}-17 \cdot \mathrm{P} 4$ fused to $\mathrm{H} 7 \cdot \mathrm{P} 4$ and $\mathrm{HP}-24 \cdot \mathrm{P} 4$ fused to $\mathrm{P} 4 \cdot \mathrm{P} 44$.

The remaining six fusion classes, however, are more difficult to determine. Since these loci have not been previously studied, no tester material is available for comparison. In addition, since three loci are involved and six genotypes are unknown, arbitrary assignment of alleles cannot be made as was done for the $Z l$ and $z L$ testers. A traditional method to analyse these $\operatorname{six} A^{2}$ tester clones would be to cross each tester to a clone of known genotype and then assign genotypes to the progeny. This would involve tedious isolation of new clones and entails numerous fusion tests. A much more efficient method of genetic analysis is shown in table 2. If these $A^{2}$ tester clones are crossed to $6 A^{4 m-1}$ clones of genotype $W X y, W x Y, w X Y$, $W x y, w X y$ and $w x Y$ but with $Q U Z L$, the phenotype of the resulting plasmodia can be found in table 2. They are all dominent at $Q U Z L$. If the plasmodia within a column were test fused among themselves, the resulting number of fusion classes and their phenotypes could be determined as shown below each column. This table can be utilised in a variety of ways to determine the $W X Y$ loci. A search was made for $A^{4 m-1}$ clones with $Q U Z L$ and $W X y, W x Y, w X Y, W x y, w X y$ and $w x Y$ genotypes. The $\mathrm{F}_{1}$ clones of fusion class (1) all have $Q U Z L$ and vary at $W X Y$.

Tests can be run to determine if the $\mathrm{F}_{1}$ clones of this fusion class are $W X Y$ or $w x y$, since fusion classes 17 and 24 have already been identified. 
Clone HP-235 of fusion class (1) was known to have the desired $Q U Z L$ genotype. In addition, tests showed that it was neither $W X Y$ nor $w x y$. In accordance with the scheme on table 2, HP-235 was crossed to the six remaining $A^{4 m-1}$ tester clones of backcross set No. 2 and then the plasmodia were test fused among themselves. Tests revealed four fusion classes, two classes with two plasmodia each and two classes with one plasmodium each.

TABLE 2

Determination of alleles at loci, $\mathrm{W}, \mathrm{X}$ and $\mathrm{Y}$ in $\mathrm{A}^{2}$ tester clones

\begin{tabular}{|c|c|c|c|c|c|c|c|}
\hline \multicolumn{2}{|c|}{$A^{2}$ Tester clones } & \multicolumn{6}{|c|}{$A^{4} m-1$ Clones from class (1) $W$ ? $X$ ? $Y$ ? } \\
\hline Class No. & Genotype & $W X y$ & $W x r$ & $w X r$ & $W x y$ & $w X y$ & $w \times r$ \\
\hline (18) & $W X y$ & $W X y$ & $W X r$ & $W X r$ & $W X y$ & $W X y$ & $W X r$ \\
\hline (19) & $w x r$ & $W X r$ & $W_{x} r$ & $W X r$ & $W \times r$ & $W X r$ & $W x r$ \\
\hline (20) & $W x y$ & $W X y$ & $W x r$ & $W X r$ & $W x y$ & $W X y$ & $W x r$ \\
\hline (21) & $w X r$ & $W X r$ & $W X r$ & $w X r$ & $W X r$ & $w X r$ & $w X r$ \\
\hline (22) & $w X y$ & $W X y$ & $W X r$ & $w X r$ & $W X y$ & $w X y$ & $w X r$ \\
\hline \multirow[t]{7}{*}{ (23) } & $w x r$ & $W X r$ & $W \times r$ & $w X r$ & $w x r$ & $w X r$ & $w \times r$ \\
\hline & & 2 fusion & 2 fusion & 2 fusion & 4 fusion & 4 fusion & 4 fusion \\
\hline & & classes & classes & classes & classes & classes & classes \\
\hline & & 3- $W X r$ & 3. $W X r$ & 3- $W X r$ & $1-W X r$ & $1-W X r$ & $1-W X r$ \\
\hline & & 3- $W X y$ & $3-W \times r$ & $3-w X Y$ & $2-W X y$ & $2-W X y$ & $2-W x r$ \\
\hline & & & & & $2-W x r$ & $2-w \times r$ & $2-w X Y$ \\
\hline & & & & & $1-W x y$ & $1-w X y$ & $1-w \times r$ \\
\hline
\end{tabular}

From table 2 it can be seen that clone HP-235 must have two recessive and one dominant fusion allele. Since none of the $W X Y$ loci has been specified HP-235 can arbitrarily be given the genotype $w \times Y$ (table 2 column at the extreme right).

Identification of the six remaining unidentified $A^{2}$ tester clones of backcross set No. 2 can now be made with the aid of information from this column. The phenotypes of the two classes with one plasmodium each should be $W X Y$ and $w \times Y$. These two plasmodia were each test fused against $\mathrm{H} 7 \cdot \mathrm{P} 4$, phenotype $Q U Z L W X Y$. One plasmodium, named $\mathrm{HP}-18 \cdot \mathrm{HP}-235$, was found to fuse to H7 $\cdot \mathrm{P} 4$. Since HP-235 is all dominant except for $w x$, in order to get a dominant phenotype at $W X$, clone HP-18 must be $W X$. Since earlier tests showed HP-18 was neither all dominant nor all recessive at $W X Y$, clone HP-18 must have at most two dominants or two recessives, therefore $\mathrm{HP}-18$ is $W X y$. The remaining plasmodium $(\mathrm{HP}-23 \cdot \mathrm{HP}-235)$ did not fuse to $\mathrm{P} 4 \cdot \mathrm{H} 7$, its phenotype is $w x Y$. Since HP-235 is $w x, \mathrm{HP}-23$ must also be $w x$. By similar reasoning as used for HP-18, HP-23 must be $w \times r$.

The phenotypes of the two classes with two plasmodia each should be $W \times Y$ and $w X Y$. These can arbitrarily be set since loci $W$ and $X$ have not yet been specified. The phenotype of plasmodia HP-19.HP-235 and HP-20.HP-235 was set at $W \times r$. From table 2 , phenotype $W \times Y$ is obtained from the following genotypes:

$$
\frac{\mathrm{HP}-235}{\mathrm{HP}(--)}=\frac{w}{W} \frac{x}{x} \frac{r}{r} \text { and } \frac{\mathrm{HP}-235}{\mathrm{HP}(--)}=\frac{w}{W} \frac{x}{x} \frac{r}{y}
$$

Therefore the two tester clones in question must have genotypes of $W \times r$ and $W x y$. To determine which clone has which genotype, HP-19 and HP-20 were each crossed to P4 and test fused to HP-20 $\cdot$ HP-235 (phenotype 
$W \times Y$ ). (Note: either HP-19 or HP-20 can be used, i.e. HP-19-HP-235 or HP-20.HP-235 since the $Y$ of HP-235 prevents phenotype variation at this locus.) Since $\mathrm{P} 4$ is dominant at all loci except $W X \Upsilon$, crossing HP-19 and HP-20 to P4 would produce plasmodia showing only the $w x y$ alleles of HP-19 and HP-20. Plasmodium HP-19 • P4 fused to HP-20 - HP-235, hence HP-19 is $W x Y$. By elimination and lack of fusion, clone HP-20 was shown to be $W x y$.

The phenotype of the remaining fusion class formed by HP-21 $\cdot$ HP-235 and HP-22-HP-235 is $w X Y$. Similarly from table 2 , it can be seen that the phenotype $w X Y$ was formed by crossing the clone $w x Y$ (HP-235) with $w X Y$ or with $w X y$. Therefore the genotype of HP-21 and HP-22 must be either $w X Y$ or $w X y$. To determine which clone has which genotype, HP-21 and HP-22 were crossed to P4 and test fused to HP-22 - HP-235 (phenotype $w X Y$ ). Plasmodium HP-21 $\cdot \mathrm{P} 4$ fused to HP-22 $\cdot \mathrm{HP}-235$ but HP-22.P4 did not. Therefore HP-21 is $w X Y$ and HP-22 is $w X y$.

It has been shown, therefore, that even though clone HP-235 was genetically undefined at loci $W, X$ and $Y$, this clone can still be used in crosses to determine the alleles at loci $W, X$ and $Y$ in the $A^{2}$ tester clones and rapid analysis of three loci simultaneously was thus possible.

\section{(iv) Physiological activity of the fusion alleles}

As was previously mentioned some of the fusion loci (weak loci) allowed temporary fusions and the production of clear zones, while other loci (strong loci) appeared to prevent any cytoplasmic mixing between two plasmodia. Once tester clones of known fusion genotype were identified, studies could be done to determine the activity of each fusion locus taken one at a time. The test to determine the function of the $Q$ locus will illustrate this process.

A plasmodium No. 1

$$
\text { (genotype } \frac{Q}{Q} \frac{R}{R} \frac{S}{S} \frac{T}{T} \frac{U V}{U V} \frac{w}{w} \frac{x}{x} \frac{y}{y} \frac{Z}{Z} \frac{L}{L} \text { ) }
$$

is test fused against a second plasmodium No. 2

$$
\text { (genotype } \frac{q}{q} \frac{R}{R} \frac{S}{S} \frac{T}{T} \frac{U V}{U V} \frac{w}{w} \frac{x}{x} \frac{y}{y} \frac{Z}{Z} \frac{L}{L} \text { ). }
$$

They differ phenotypically only at the $Q$ locus. Each test fusion was run in at least 10 replicates. The two plasmodia are observed continually for 3 minutes after they meet in an effort to detect any temporary fusions and development of clear zones. If no interactions are observed, the plasmodia are again observed once after 12 minutes. Observations were made at $45 \times$ in a dissecting microscope. Based on these tests fusion loci $Q, R, S, T, U, V$, and $Y$ were determined to be strong loci; the plasmodia used in these tests did not fuse even temporarily. Loci $W, X, Z$ and $L$ were determined to be weak loci; the plasmodia used in these tests fused temporarily and later clear zones were formed.

The plasmodia that did not appear to fuse at all were tested again at $150 \times$ in a compound microscope. Fusion tests were again observed continuously for at least 3 minutes with 10 replicates for each of the seven loci tested. Again no fusions were observed. Based on these results we suggest a revision of the plasmodial fusion system nomenclature as shown in table 3 . 
Fig. 2.-A typical non-fusion. A distinct separation can be seen between the plasmodium on the top and the one on the bottom. $\times 17$.

Fig. 3.-A typical fusion. Several veins of streaming protoplasm connect the plasmodium on the top to the one on the bottom. $\times 17$.

Fig. 4.-A typical clear zone (at the arrow). This distinct area, where protoplasmic mixing has occurred, stands out because of its different colour and texture. $\times 17$. 

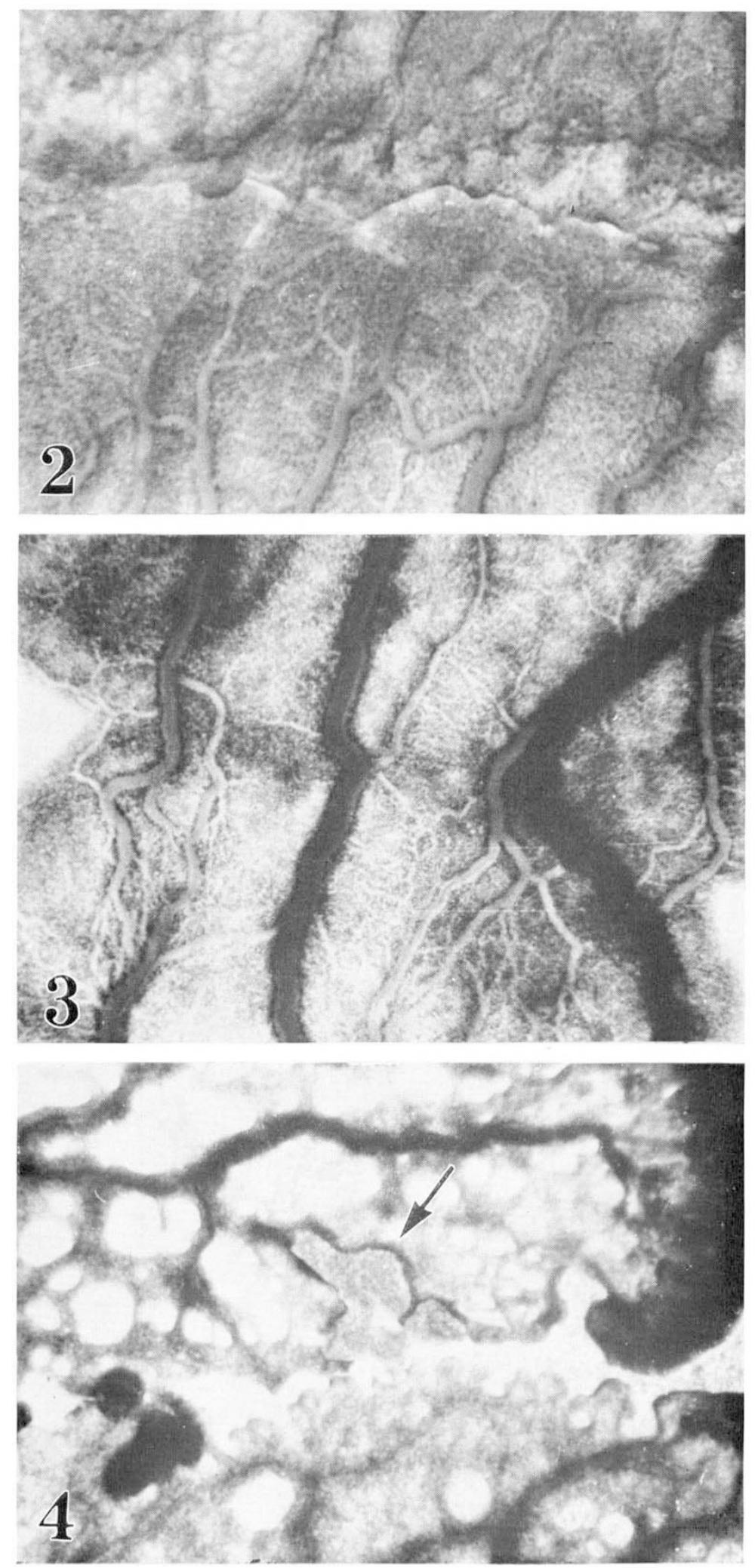

Plate I 
TABLE 3

Nomenclature of the fusion loci

$\begin{array}{cc}\text { Old designations } & \text { New designations } \\ Q, q & \text { Fus } 1, \text { fus } 1 \\ R, r & \text { Fus } 2, \text { fus } 2 \\ S, s & \text { Fus } 3, \text { fus } 3 \\ T, t & \text { Fus } 4, \text { fus } 4 \\ U, u & \text { Fus } 5, \text { fus } 5 \\ V, v & \text { Fus } 6, \text { fus } 6 \\ W, w & \text { Cz1, cz1 } \\ X, x & \text { Cz2, cz2 } \\ r, y & \text { Fus } 7, \text { fus } 7 \\ Z, z & C z 3, c z 3 \\ L, l & C z 4, c z 4\end{array}$

\section{Discussion}

We currently have very little biochemical data on how cell fusion is controlled. From analogy, however, with other systems of cell recognition, it appears quite possible that fusion may be controlled by specific gene determinants (protein, glycoproteins, etc.) on the plasma membrane. The gene product of the strong fusion loci would thus give each plasmodium its biochemical uniqueness (cf. Reisfeld and Kahan, 1970). The weak loci may control cytoplasmic determinants which recognise other compounds in foreign cytoplasm. Such recognition may be similar to mating system recognition in protists or antibody-antigen reactions in vertebrates. We believe that these postulated weak loci determinants must be constitutive and not be dependent on an inducing mechanism since coagulation reactions forming clear zones may occur in as little as 30 seconds.

The plasmodial fusion system outlined here appears to have several similarities to the histocompatibility system in mammals. Both systems deal with mechanisms which distinguish self from non-self; both systems are controlled by numerous independent loci and in both systems there exist strong and weak loci.

It is interesting to speculate that a cellular defence system similar to that found in plasmodia eventually evolved into the complex immunological system found in vertebrates. The biochemical relationships, if any, between the plasmodial fusion system and defence and recognition systems in higher plants and animals await further research (cf. Klement and Goodman, 1967).

Acknowledgment.-We gratefully acknowledge support by the Research Corporation (Brown Hazen Fund), the University of Delaware Research Foundation, and PHS grant GM-19-16501. We are also indebted to Andrew Liu for his competent assistance.

\section{REFERENGES}

Alexopoulos, C. J., AND ZABKA, G. G. 1962. Production of hybrids between physiological races of the true slime mold Didymium iridis. Nature, 193, 598-599.

Bernet, J. 1967. Les systèmes d'incompatibilité chez le Podospora anserina. C.R. Acad. Sci. Paris, 265, 1330-1333.

CATEN, C. E. 1971. Heterokaryon incompatibility in imperfect species of Aspergillus. Heredity, 26, 299-312. 
CoLLINs, o. R. 1963. Multiple alleles at the incompatibility locus in the myxomycete Didymium iridis. Amer. F. Bot., 50, 477-480.

COLLINS, O. R., AND CLARK, J. 1966. On the genetic basis of plasmodial compatibility in Didymium iridis. Amer. F. Bot., 53, 625.

COLLINS, O. R., AND CLARK, J. 1968. Genetics of plasmodial compatibility and heterokaryosis in Didymium iridis. Mycologia, 60, 90-103.

COLLINS, O. R., AND CLARK, J. 1973. Directional cytotoxic reactions between compatible plasmodia of Didymium iridis. Genetics, 73, 247-257.

COLLINS, O. R., AND LING, H. 1964. Further studies in multiple allelomorph heterothallism in the myxomycete Didymium iridis. Amer. 7. Bot., 51, 315-317.

COLLINS, O. R., AND LING, H. 1972. Genetics of somatic cell fusion in two isolates of Didymium iridis. Amer. 7. Bot., 59, 337-340.

FINCHAM, J. R. s., AND DAY, P. R. 1971. Fungal Genetics, Third Ed. Blackwell Scientific Publications, Oxford.

HARRIs, H. 1970. Cell Fusion. Harvard University Press, Cambridge.

JONEs, D. A. 1965. Heterokaryon incompatibility in the Aspergillus glaucus Link group. Heredity, 20, 49-56.

KLEMENT, K., AND GOODMAN, R. N. 1967. The hypersensitive reaction to infection by bacterial plant pathogens. Ann. Rev. Phytopathol., 5, 17-44.

LING, H. 1971a. Control of plasmodial fusion in a Panamanian isolate of Didymium iridis. Diss. Abst. Int'l., 32, 2558-B.

LING, H. 1971 $b$. Genetics of somatic fusion, in a myxomycete; $\mathrm{F}_{2}$ studies. Protoplasma, 73, $407-416$

LING, H., AND COllins, O. R. 1970a. Control of plasmodial fusion in a Panamanian isolate of Didymium iridis. Amer. F. Bot., 57, 292-298.

LING, H., AND COLLINs, O. R. 1970b. Linkage studies in the true slime mold Didymium iridis. Amer. 7. Bot., 57, 299-303.

MUKHERJEE, K. L. 1965. Plasmodial fusion reactions between three races of Didymium iridis and their hybrids. 7. Indian Bot. Soc., 44, 224-230.

POOLE, A. R., HOWELL, J. I., AND LUCY, J. A. 1970. Lysolecithin and cell fusion. Nature, 227, 810-814.

POWER, J. B., CUMMINS, S. E., AND COCKING, E. C. 1970. Fusion of isolated plant protoplasts. Nature, 225, 1016-1018.

REISFELD, R. A., AND KAHAN, B. D. 1970. Transplantation antigens. Advan. Immunol., 12, $117-200$.

UPADHYAYA, K. C., AND LING, H. 1972. Fine structure of clear zones produced by partial intermixing of protoplasm of two phenotypically different plasmodia in a myxomycete. Amer. 7. Bot., 59, 668.

WILsON, J. F., AND GARNJOBst, L. 1966. A new compatibility locus in Neurospora crassa. Genetics, 53, 621-631. 